\title{
A New Way to Age Estimation for RGB-D Images, based on a New Face Detection and Extraction Method for Depth Images
}

\author{
Seyed Muhammad Hossein Mousavi \\ Bu Ali Sina University / Department of Computer Engineering, Hamadan, 65141, Iran \\ Email: mosavi.a.i.buali@gmail.com, h.mosavi93@basu.ac.ir
}

Received: 18 August 2018; Accepted: 22 September 2018; Published: 08 November 2018

\begin{abstract}
With adding depth data to color data, it is possible to increase recognition accuracy significantly. Depth image mostly uses for calculating range or distance between object and sensor. Also they are used for making 3-D models of objects and increasing accuracy. Depending on the sensor's depth quality, the recognition accuracy changes. Age estimation is useful for calculating the aging effects using prior patterns, which are recorded during years from subjects. In this paper, age estimation occurs using summation of RGB image edges gray value and summation of depth image's entropy edges. Furthermore, a new face detection and extraction method for depth images is represented, which is based on standard deviation filter, ellipse fitting and some prepost processing techniques. The advantage of this method is its speed and single image aspect capability. In this approach, there is no need to learning and classification process. Proposed method is between 10 to 20 times faster but lower accurate. System is validated with some benchmark color and color-depth (RGB-D) face databases, and in comparing with other age estimation methods, returned satisfactory and promising results. Because of the high speed in this method, it is possible to use it on real time applications. It is mentionable that this paper is the first age estimation research on RGB-D images.
\end{abstract}

Index Terms-Depth image, Age estimation, Depth sensor, RGB-D image, Benchmark database, High speed

\section{INTRODUCTION}

Aging effect, Influences our life aspects during time. With having right knowledge about this phenomenon, we can manage it properly for the rest of our lives. But how much is the effect value of this phenomenon? Using image processing techniques, it is possible to answer this question. Automatic age estimation is very useful in medicine, psychology, 3D modeling, soft biometrics [2], human-computer interaction (HCI) [1], security control [2], surveillance monitoring [3], and electronic customer relationship management [2]. This section pays to some of the basic definitions of the subject. Sections II, III, IV and $\mathrm{V}$ respectively represented, some prior works on the subject by other researchers until 2017, our proposed method on age estimation using color-depth (RGB-D) images and proposed face detection and extraction method for depth images, evaluation results on three benchmark face databases and finally conclusion and suggestion on subject for improving the proposed method.

\section{A. Image processing in pattern recognition}

Using of computer algorithms for manipulating digital images is called image processing. Digital image processing focuses on two major tasks [4]:

- Improvement of pictorial information for human interpretation

- Processing of image data for storage, transmission and representation for autonomous machine perception

Pattern recognition is the process of classifying input data into objects or classes based on key features. There are two classification methods in pattern recognition: supervised and unsupervised classification. Pattern recognition has applications in computer vision, radar processing, speech recognition, and text classification [5] [6]. Changing, learning, improving and manipulating digital image based on some patterns by computer algorithms called image processing in pattern recognition.

\section{B. Age estimation}

Aging effect will determine by gender and race, and it is distinct in different weather, and also it is depended on other factors like job, family environment, diet, sicknesses and hereditary effects. So we can't say, "age detection" and should use "age estimation", because it is not a precise subject. The only way that we can use age detection, is when using the face patterns during a person's life time, and that is just belonged to that specific person. However, using image processing techniques, there is high possibility to estimate different race people ages. With extracting proper patterns from digital image and using appropriate pre and post processing methods, age estimation is possible with good accuracy. Figure 1 
represents the aging effect on two people from the FGNet aging database [8] in different ages [7].

\section{Depth image}

Depth images mostly are using for range and distance detection. There are several depth sensors, which most of them use infrared spectrum to sense the distance between the object and the sensor. Kinect is one of the most popular depth sensors in the world, especially among researchers. For more information about Kinect sensors it can be referred to [9]. Depth images are so popular in 3D modeling in making animation and--games [10], HumanComputer Interaction (HCI) [11], industry [12], robotics [13] and more. Here we are using it for increasing the accuracy of age estimation along with color image.

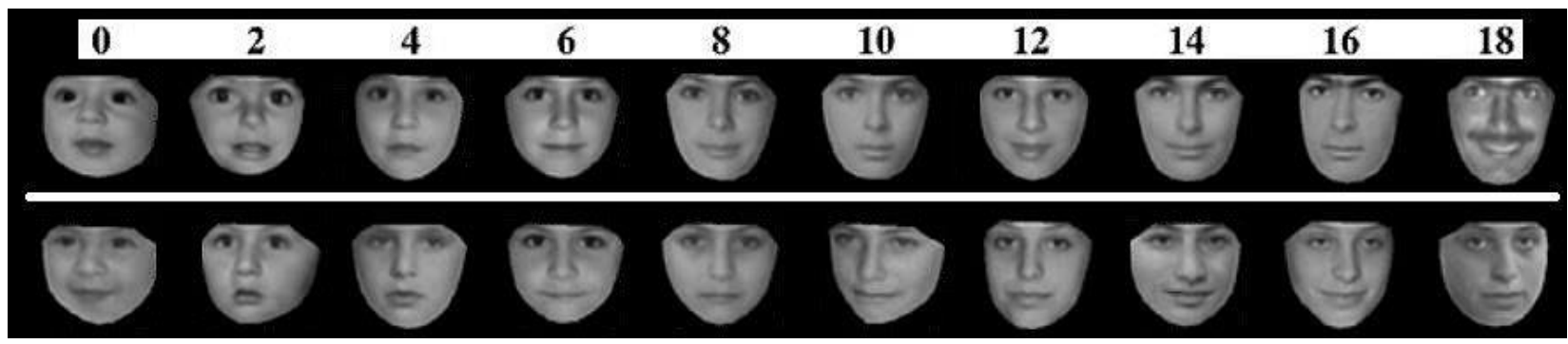

Fig.1. Aging effect on two persons from the FG-Net aging database [8] in different ages [7]

\section{Face detection and extraction}

The process of distinguishing a face object from other objects in the image is called face detection. After face detection process and for working on just face object, it is needed to extract the face, which it is possible by cropping technique. There are diverse face detection methods like ellipse fitting or viola and Jones face detection algorithm, which in this paper we used from viola and Jones algorithm for face detection of color images. This algorithm is demonstrated in detailed in section 3. Figure 2 represents some examples of face detection from RGB and Depth images [14, 15]. Face detection is first part of face recognition, gender recognition, facial expression recognition and other like activities.

\section{RELATED WORKS}

Age estimation and face detection are active field in image processing. Because of the lack of space and better readability of the paper, History and prior works on age estimation and face detection from last decade, until now, is listed in Table 1. Despite of Table 1, there are mentionable researches on automatic age estimation and face detection purposes such as Geng, Xin, et al, work in 2006 on AGES (AGing pattErn Subspace) method for automatic age estimation. The basic idea was to model the aging pattern, which is defined as a sequence of personal aging face images, by learning a representative [35]. Also, Hayashi, Jun-ichiro, et al, succeeded to make a system for age and gender estimation based on wrinkle texture and color of facial images in 2002 [36]. Also [37, 38,39 ] researches on age estimation using color images are so valuable. Yang, Guangzheng, and Thomas S. Huang made a new method to locate human faces in a complex background in 1994 [40]. The system could locate unknown human faces spanning a wide range of sizes in a complex black-and-white picture. In 2000, Wang, Jianguo, and Tieniu Tan made a system for face detection based on shape informations [41]. Using fuzzy theory in face detection was Wu, Haiyuan, Qian Chen's idea in 1999 [42]. A great survey on face detection algorithms could be found in [43].

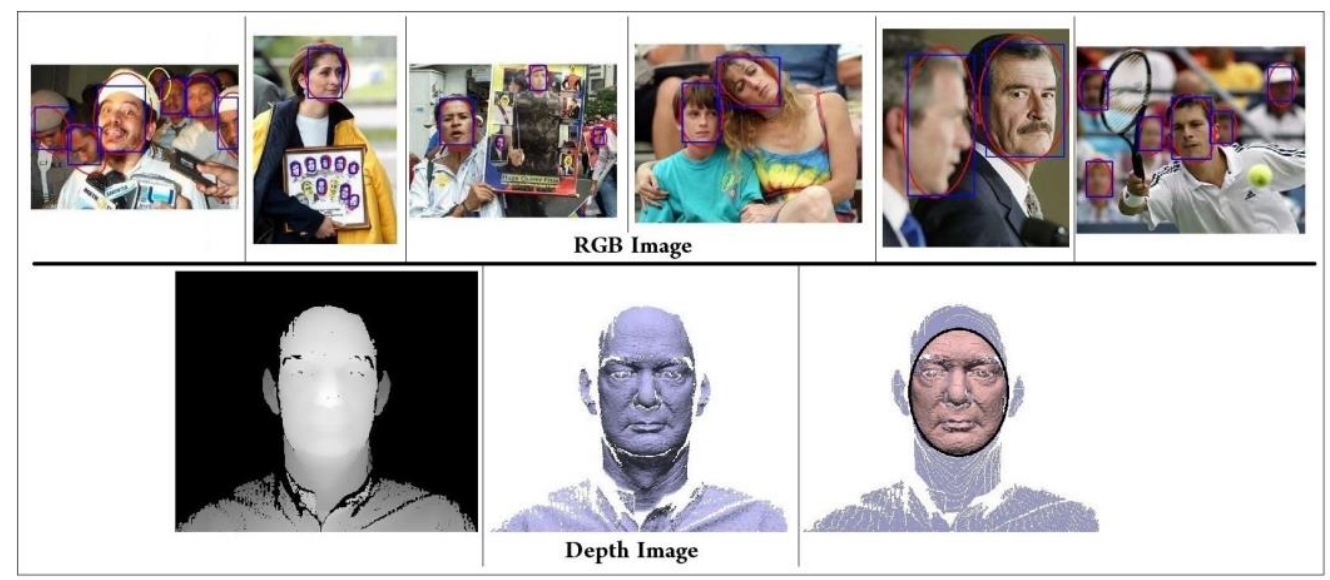

Fig.2. Examples of face detection from RGB and Depth images [14, 15] 
Table 1. Some of the prior researches on age estimation and face detection algorithms in last decade

\begin{tabular}{|c|c|c|c|c|c|c|}
\hline Function & Features and descriptions & Classifier & Database & Accuracy & Ref & Cite \\
\hline Age Estimation & PCA & SVM & FG-NET & $\begin{array}{c}\text { Mean } \\
\text { Absolute Error } \\
\text { (MAE): } 6.77\end{array}$ & [16] & 728 \\
\hline Age Estimation & $\begin{array}{c}\text { Patch-based Gaussian Mixture Models } \\
\text { (GMM) and Hidden Markov Models } \\
\text { (HMM) Supervector }\end{array}$ & $\begin{array}{l}\text { Nearest } \\
\text { Centroid } \\
\text { Classifier } \\
\text { (NCC) }\end{array}$ & Novel & $\begin{array}{l}\text { MAE: } \\
\text { Male }=5.397 \\
\text { Female }= \\
6.333\end{array}$ & [17] & 49 \\
\hline Age Estimation & $\begin{array}{c}\text { Preprocessing }=\text { Active Appearance Model } \\
\text { (AAM) } \\
\text { Feature }= \\
\text { Least Angle Regression (LAR) }\end{array}$ & $\begin{array}{l}\text { Support } \\
\text { Vector } \\
\text { Regression } \\
\text { (SVR) }\end{array}$ & FG-NET & MAE: 5.70 & {$[18]$} & 43 \\
\hline Age Estimation & \multicolumn{2}{|c|}{$\begin{array}{l}\text { Ordinal discriminative aging features and Preserving } \\
\text { Locality and Ordinal Information (PLO) method }\end{array}$} & FG-NET & MAE: 4.82 & [19] & 90 \\
\hline Age Estimation & $\begin{array}{l}\text { Facial Component Localization using } \\
\text { Active Shape Model (ASM) and } \\
\text { Biologically Inspired Feature (BIF) }\end{array}$ & SVM & $\begin{array}{l}\text { FG-NET } \\
\text { PCSO }\end{array}$ & $\begin{array}{l}\text { MAE: } 4.7 \\
\text { MAE: } 7.2\end{array}$ & {$[20]$} & 115 \\
\hline Age Estimation & \multicolumn{2}{|l|}{ convolutional neural network $(\mathrm{CNN})$} & MORPH & MAE: 3.63 & [21] & 56 \\
\hline Age Estimation & \multicolumn{2}{|l|}{ convolutional neural network $(\mathrm{CNN})$} & Novel & $84.7 \%+,-2.2$ & [22] & 211 \\
\hline Age Estimation & \multicolumn{2}{|c|}{ convolutional neural network (CNN) } & $\begin{array}{l}\text { MORPH } \\
\text { AFAD }\end{array}$ & $\begin{array}{l}\text { MAE: } 3.27 \pm \\
0.02 \\
\text { MAE: } 3.34 \pm \\
0.08\end{array}$ & [23] & 30 \\
\hline Age Estimation & \multicolumn{2}{|c|}{ group-aware deep feature learning (GA-DFL) } & $\begin{array}{l}\text { FG-NET } \\
\text { MORPH } \\
\text { Chalearn- } \\
\text { Challenge }\end{array}$ & $\begin{array}{l}\text { MAE: } 3.93 \\
\text { MAE: } 3.25 \\
\text { MAE: } 4.21\end{array}$ & [24] & 6 \\
\hline Face Detection & \multicolumn{4}{|c|}{ RGB images (Weak in Depth images) } & [25] & 16250 \\
\hline Face Detection & \multicolumn{4}{|c|}{ RGB images (Using Ellipse fitting in the paper, page 6) } & [26] & 23 \\
\hline Face Detection & \multicolumn{4}{|c|}{ Depth images } & [27] & 22 \\
\hline
\end{tabular}

\section{PROPOSED METHOD}

This section pays to the important algorithms made by other researchers and proposed method's procedure.

\section{A. Viola and jones face detection algorithm}

Viola and Jones algorithm is one of the best face detection algorithms, which is using for a long period of time in face image processing. This algorithm is so fast and robust, and it could be used for depth images too, but with lower accuracy. This algorithm is an object detection algorithm and could be used for any learned object but mainly uses for face. The algorithm has four stages:

1. Haar Feature Selection

2. Creating an Integral Image

3. Adaboost Training

4. Cascading Classifiers
5. After detecting face by this algorithm, simply it can be extracted by cropping technique. For more information and details about this algorithm could refer to [25].

\section{B. Proposed face detection and extraction from depth image}

There are a lot of face detection algorithms for color or RGB images, but there are few face detection algorithms for depth images like [27]. So it is decided to make a fast, precise and robust face detection and extraction algorithm for depth images. The main body of the processes is to find the closest pixel to the sensor (nose tip) and using the standard deviation filter [28] and ellipse fitting [29] techniques on depth image. The complete procedure of proposed face detection method is demonstrated in Figure 3. 


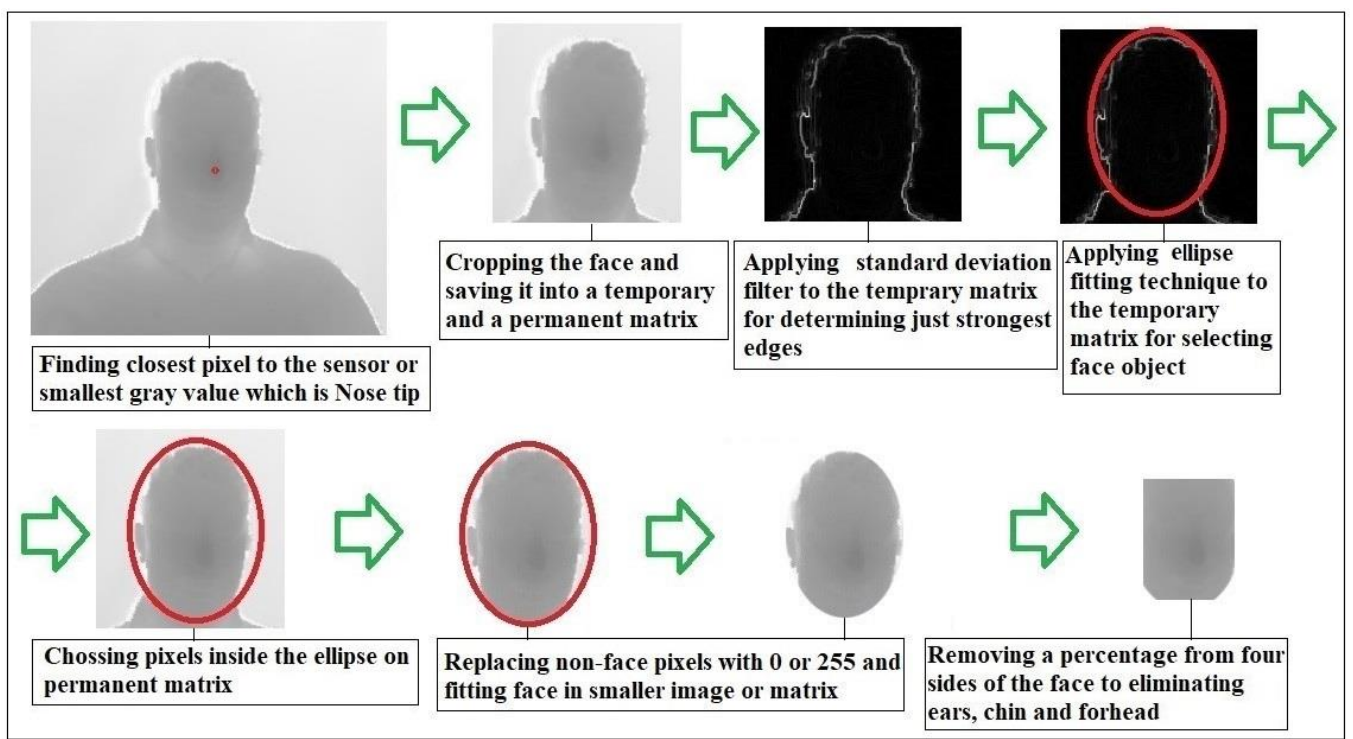

Fig.3. Proposed face detection and extraction method's workflow out of depth data

\section{Image entropy}

Entropy is a statistical measure of randomness that can be used to characterize the texture of the input image. Image entropy is a quantity which is used to describe the business of an image, i.e. the amount of information which must be coded for by a compression algorithm. An image that is perfectly flat will have an entropy of zero. Consequently, they can be compressed to a relatively small size. On the other hand, high entropy images such as an image of heavily cratered areas on the moon have a great deal of contrast from one pixel to the next and consequently, cannot be compressed as much as low entropy images [4]. Image entropy formula is as (1):

$$
\text { Entropy }=-\sum P_{i} \log _{2} P^{i}
$$

In the above expression, $\mathrm{Pi}$ is the probability that the difference between two adjacent pixels is equal to $\mathrm{i}$, and $\log 2$ is the base 2 logarithm [30].

\section{Proposed age estimation method}

After face detection and extraction for color images by viola and Jones algorithms and the same procedure for depth images by proposed face detection and extraction method from depth images, it is time to implement proposed age estimation method on the preprocessed images of databases. The main idea is to use a specific filter, for color and entropy filter for depth images and sum of the gray-scale pixels for final normalization. Proposed edge detection filter is clear in Table 2. The complete procedure of proposed method is shown in Figure 4. It is clear that for the color image from FG-NET database, just first row of the process will be done (due to having just color data). Figure 5 presents the proposed method's flowchart to estimating age.

Table 2. Proposed edge detection filter

\begin{tabular}{|l|l|l|l|l|l|}
\hline-1.2 & -0.8 & -0.6 & 1.2 & 0.8 & 0.6 \\
\hline 0 & 0 & 0 & 0 & 0 & 0 \\
\hline 1.2 & 0.8 & 0.6 & -1.2 & -0.8 & -0.6 \\
\hline 1.2 & 0 & -1.2 & -1.2 & 0 & 1.2 \\
\hline 0.8 & 0 & -0.8 & -0.8 & 0 & 0.8 \\
\hline 0.6 & 0 & -0.6 & -0.6 & 0 & 0.6 \\
\hline
\end{tabular}




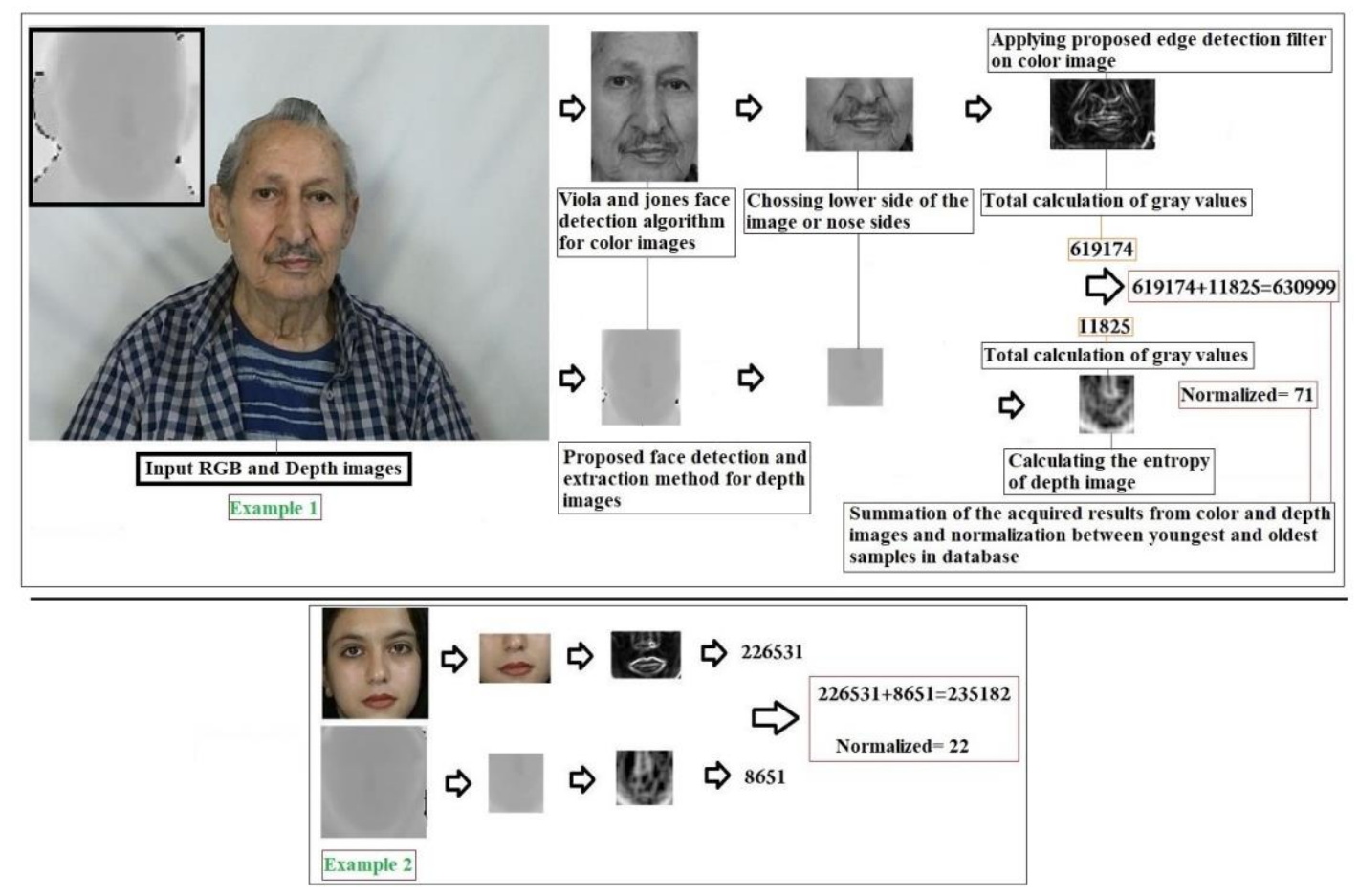

Fig. 4. Proposed age estimation method's workflow

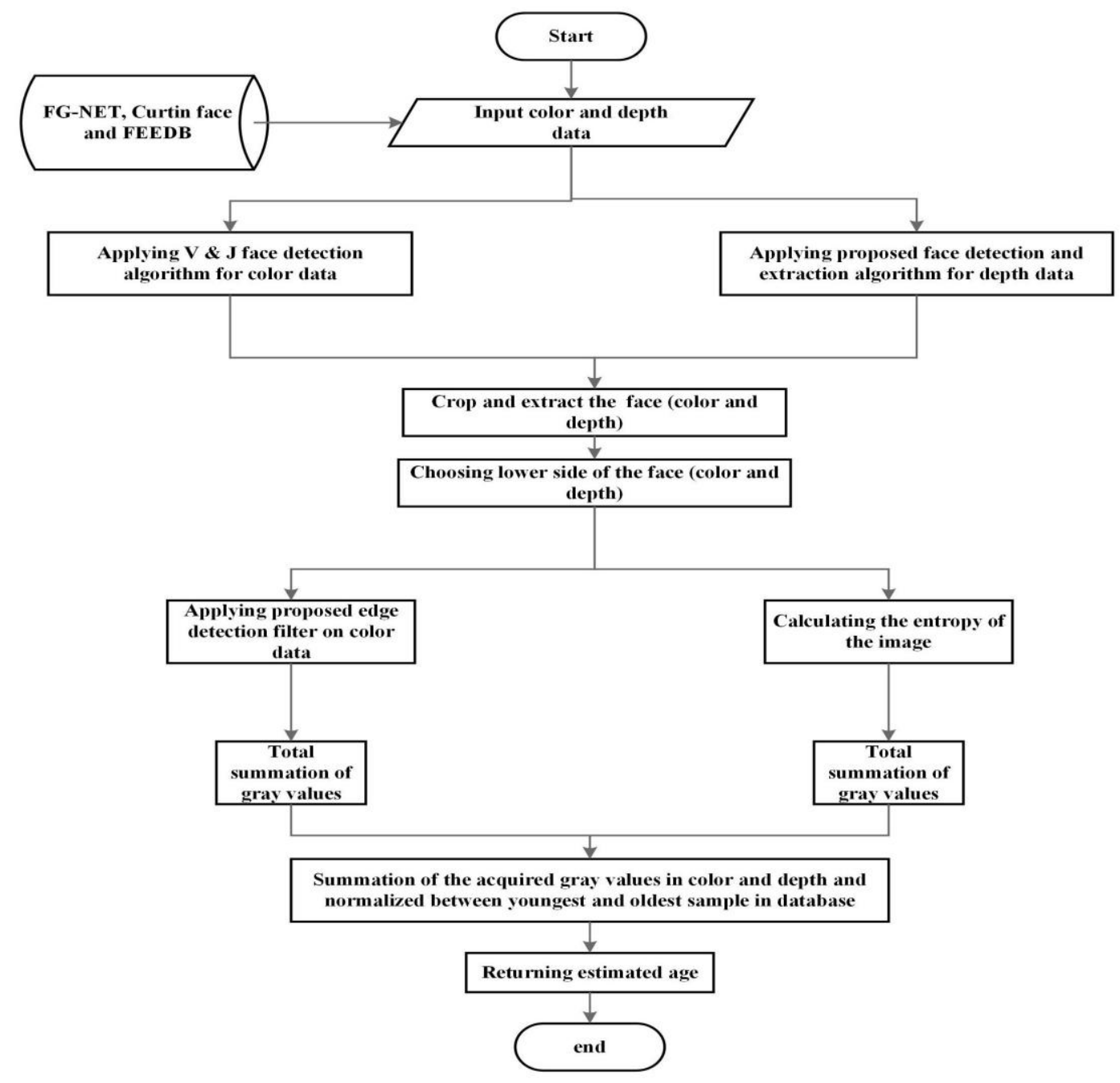

Fig.5. The proposed method's flowchart to estimating age 


\section{TEST AND VALIDATION}

In this section, proposed method is tested and validated by Mean Absolute Error (MAE) [31] factor on three RGB and RGB-D databases, which are FG-NET [8], Curtin face [32] and FEEDB [33]. The evaluations are done for all age ranges and in four ranks. In addition, these evaluations are done for male, female and both genders respectively.
Figure 6 shows some of the samples from FG-NET, Curtin face and FEEDB databases. Table 4 shows the evaluations results.

\section{A. Databases}

We used three face databases for evaluation, which one of them was a color database and other two were RGB-D type. The complete information about databases is listed in Table 3.

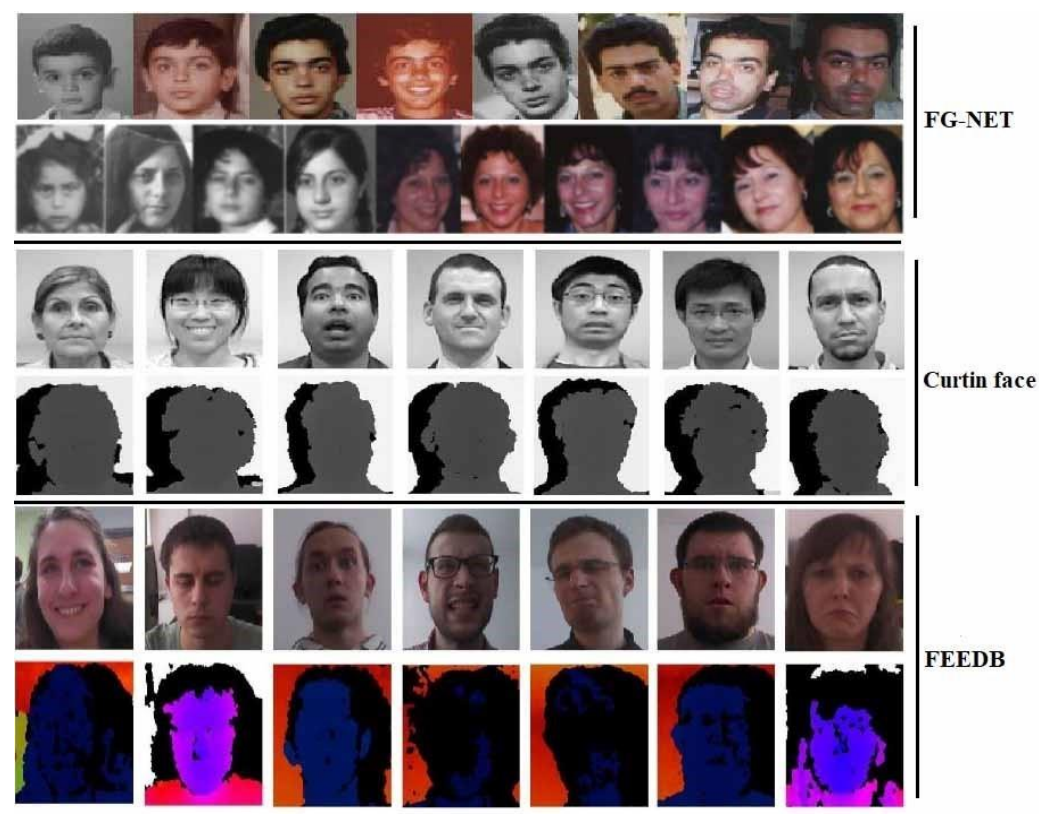

Fig.6. Some of the samples from FG-NET, Curtin face and FEEDB databases

Table 3. Used databases details

\begin{tabular}{|c|c|c|c|}
\hline Database & FG-NET & Curtin face & FEEDB \\
\hline Authors & FG-NET Group & Li, Billy YL, et al. & Szwoch, Mariusz \\
\hline Samples & 82 & 52 & 50 \\
\hline Sensor & Different types & Kinect V.1 & Kinect V.1 \\
\hline Resolution & $\begin{array}{l}\text { Different resolutions and } \\
\text { quality }\end{array}$ & $\begin{array}{l}\mathrm{RGB}=640 * 480 \\
\mathrm{Depth}=640 * 480\end{array}$ & $\begin{array}{l}\text { RGB }=640 * 480 \\
\text { Depth }=640 * 480\end{array}$ \\
\hline Year & 2004 & 2013 & 2013 \\
\hline Size & 43 Mega byte & 12 Giga byte & Around 100 Giga byte \\
\hline Reference & {$[5]$} & {$[26]$} & {$[27]$} \\
\hline Cite & 365 till 2014 based on [28] & 150 till 2017 & 21 till 2017 \\
\hline
\end{tabular}

\section{B. Mean Absolute Error (MAE)}

Mean Absolute Error (MAE) [31] is a measure of difference between two continuous variables. Assume $\mathrm{X}$ and $\mathrm{Y}$ are variables of paired observations that express the same phenomenon. Examples of $\mathrm{Y}$ versus $\mathrm{X}$ include comparisons of predicted versus observed, subsequent time versus initial time, and one technique of measurement versus an alternative technique of measurement. Consider a scatter plot of $\mathrm{n}$ points, where point $\mathrm{i}$ has coordinates (xi, yi)... Mean Absolute Error (MAE) is the average vertical distance between each point and the $\mathrm{Y}=\mathrm{X}$ line, which is also known as the One-
to-One line. MAE is also the average horizontal distance between each point and the $\mathrm{Y}=\mathrm{X}$ line. The mean absolute error is a common measure of forecast error in the timeseries analysis, [34] where the terms "mean absolute deviation" is sometimes used in confusion with the more standard definition of mean absolute deviation. Table 4 represents our method results. Additionally, Figure 7 shows Table 4 result's in graphical chart form.

$$
M A E=\frac{\sum_{i=1}^{n}\left|y_{i}-x_{i}\right|}{n}=\frac{\sum_{i=1}^{n}\left|e_{i}\right|}{n}
$$


Table 4. Evaluation's results (MAE for each rank and database)

\begin{tabular}{|l|l|l|l|}
\hline Database & FG-NET & Curtin Face & FEEDB \\
\hline Rank 1 $\mathbf{( 2 5} \%)$ & Female: 11.2 & Female: 9.14 & Female: 14.83 \\
& Male: 9.67 & Male: 9.36 & Male: 19.11 \\
& Both: 10.43 & Both:9.25 & Both: 16.97 \\
\hline Rank 2 (50 \%) & Female: 12.8 & Female: 10.10 & Female: 16.98 \\
& Male: 12.19 & Male: 9.82 & Male: 21.24 \\
& Both: 12.49 & Both: 9.96 & Both: 19.11 \\
\hline Rank 3 (75 \%) & Female: 15.9 & Female: 11.99 & Female: 18.37 \\
& Male: 13.22 & Male: 11.67 & Male: 25.44 \\
& Both: 14.56 & Both: 11.83 & Both: 21.90 \\
\hline Rank 4 (100 \%) & Female: 19.40 & Female: 13.20 & Female: 26.95 \\
& Male: 21.7 & Male: 12.18 & Male: 31.03 \\
& Both: 20.55 & Both: 12.69 & Both: 28.99 \\
\hline
\end{tabular}

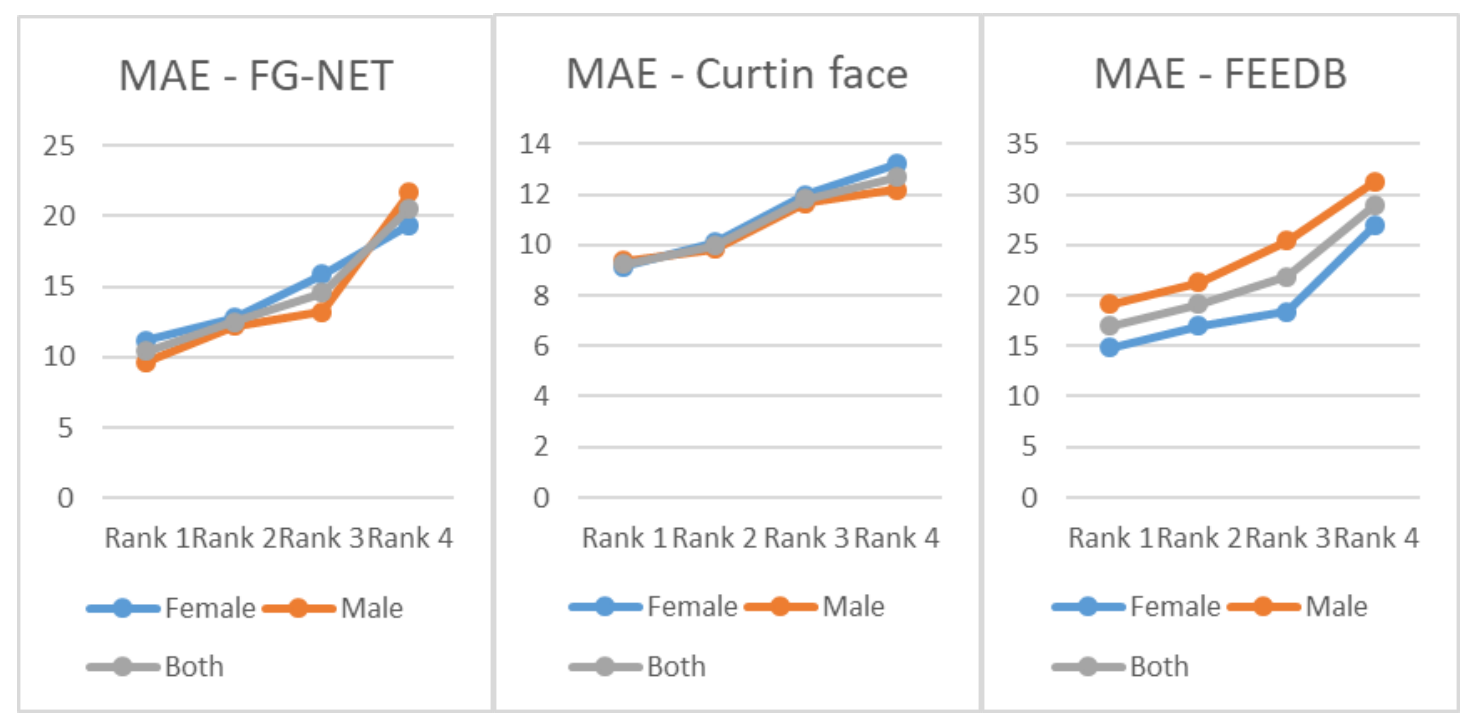

Fig.7. Table 4 result's in graphical chart form

\section{Evaluations results and discussion}

As it mentioned before, because of our singled image age estimation method, there is no need to have a different image in different ages from a subject, and using a single image could be satisfying enough. So due to high speed calculation and no need to learn, this method could be used in real time systems. But in such a condition, low accuracy could be experienced, which is normal. The results are gained from $25 \%, 50 \%, 75 \%$ and $100 \%$ of databases in male, female and all subjects' forms. We select best samples from different ages of mentioned databases and divided them into four mentioned ranks, and used MAE factor to calculate error between real and acquired ages result. The final MAE value is the average of acquired MAE values for each Female, Male and both states. It is clear, the more rank; the less accuracy and best results with low errors are belonged to first rank and worst results belonged to last rank. As it is clear, the best result with lowest MAE is belonged to rank 1 of Curtin face database with MAE of 9.25 and worst one is belonged to rank 4 of FEEDB database with MAE of 28.99. Also because of high run time speed of our method, we could not to increase FG-Net database accuracy according to other researcher's results, but in relation of our method's speed, acquired results are completely satisfactory. Also it is mentionable that age estimation process on Curtin face and FEEDB database and even on RGB-D images is done for the first time in this paper.

\section{CONCLUSION AND SUGGESTION}

In this paper, a new age estimation method based for RGB-D images is presented. Also a new method for face detection and extraction for depth images is presented, which is very robust and fast. The golden point in this paper is the high run time speed and having real time capability. Due to need for learning and even single image based ability of this system, run time speed against learning based system is 10 times better, but low accuracy is vivid. Because of the high speed of the algorithm, it is suggested to use this method on mobile phone apps. Moreover, it is suggested to use proposed method on other different color and depth database such as: MORPH, KDEF, EURECOM, VAP RGB-D and VAP RGBD-D-T.

\section{REFERENCES}

[1] Geng, Xin, Zhi-Hua Zhou, and Kate Smith-Miles. "Automatic age estimation based on facial aging patterns." IEEE Transactions on pattern analysis and machine intelligence 29.12 (2007): 2234-2240. 
[2] Y. Fu, G. Guo, and T. Huang. Age synthesis and estimation via faces: a survey. IEEE Trans. Pattern Anal. Mach. Intell., 32(11):1955-1976, 2010.

[3] Z. Song, B. Ni, D. Guo, T. Sim, and S. Yan. Learning universal multi-view age estimator by video contexts. In Proc. ICCV, 2011.

[4] Gonzalez, Rafael (2008). 'Digital Image Processing, 3rd'. Pearson Hall. ISBN 9780131687288.

[5] https://www.mathworks.com/discovery/patternrecognition.html

[6] Duda, Richard O., Peter E. Hart, and David G. Stork. Pattern classification. John Wiley \& Sons, 2012.

[7] Geng, Xin, Zhi-Hua Zhou, and Kate Smith-Miles. "Automatic age estimation based on facial aging patterns." IEEE Transactions on pattern analysis and machine intelligence 28.12 (2007): 2234-2240.

[8] The FG-NET Aging Database, http://sting.cycollege.ac.cy/ alanitis/ fgnetaging/index.htm, 2002.

[9] https://www.slideshare.net/SugiuraTsukasa/kinect-v2introduction-and-tutorial

[10] Henry, Peter, et al. "RGB-D mapping: Using Kinect-style depth cameras for dense 3D modeling of indoor environments." The International Journal of Robotics Research 31.5 (2012): 647-663.

[11] Ren, Zhou, Jingjing Meng, and Junsong Yuan. "Depth camera based hand gesture recognition and its applications in human-computer-interaction." Information, Communications and Signal Processing (ICICS) 2011 8th International Conference on. IEEE, 2011.

[12] Cruz, Leandro, Djalma Lucio, and Luiz Velho. "Kinect and rgbd images: Challenges and applications." 2012 25th SIBGRAPI Conference on Graphics, Patterns and Images Tutorials. IEEE, 2012.

[13] El-laithy, Riyad A., Jidong Huang, and Michael Yeh. "Study on the use of Microsoft Kinect for robotics applications." Position Location and Navigation Symposium (PLANS), 2012 IEEE/ION. IEEE, 2012.

[14] Sun, Xudong, Pengcheng Wu, and Steven CH Hoi. "Face detection using deep learning: An improved faster rcnn approach." arXiv preprint arXiv:1701.08289 (2017).

[15] ter Haar, Frank B., and Remco C. Veltkamp. "3D face model fitting for recognition." European Conference on Computer Vision. Springer, Berlin, Heidelberg, 2008.

[16] Geng, Xin, Zhi-Hua Zhou, and Kate Smith-Miles. "Automatic age estimation based on facial aging patterns." IEEE Transactions on pattern analysis and machine intelligence 29.12 (2007): 2234-2240.

[17] Zhuang, Xiaodan, et al. "Face age estimation using patchbased hidden markov model supervectors." Pattern Recognition, 2008. ICPR 2008. 19th International Conference on. IEEE, 2008.

[18] Ricanek, Karl, et al. "Generalized multi-ethnic face ageestimation." Biometrics: Theory, Applications, and Systems, 2009. BTAS'09. IEEE 3rd International Conference on. IEEE, 2009.

[19] Li, Changsheng, et al. "Learning ordinal discriminative features for age estimation." Computer vision and pattern recognition (cvpr), 2012 ieee conference on. IEEE, 2012.

[20] $\mathrm{Han}, \mathrm{Hu}$, Charles Otto, and Anil K. Jain. "Age estimation from face images: Human vs. machine performance." Biometrics (ICB), 2013 International Conference on. IEEE, 2013.

[21] Yi, Dong, Zhen Lei, and Stan Z. Li. "Age estimation by multi-scale convolutional network." Asian Conference on Computer Vision. Springer, Cham, 2014.
[22] Levi, Gil, and Tal Hassner. "Age and gender classification using convolutional neural networks." Proceedings of the IEEE Conference on Computer Vision and Pattern Recognition Workshops. 2015.

[23] Niu, Zhenxing, et al. "Ordinal regression with multiple output cnn for age estimation." Proceedings of the IEEE Conference on Computer Vision and Pattern Recognition. 2016.

[24] Liu, Hao, et al. "Group-aware deep feature learning for facial age estimation." Pattern Recognition 66 (2017): 8294.

[25] Viola, Paul, and Michael Jones. "Rapid object detection using a boosted cascade of simple features." Computer Vision and Pattern Recognition, 2001. CVPR 2001. Proceedings of the 2001 IEEE Computer Society Conference on. Vol. 1. IEEE, 2001.

[26] Carcagnì, Pierluigi, et al. "Facial expression recognition and histograms of oriented gradients: a comprehensive study." SpringerPlus 4.1 (2015): 645.

[27] ter Haar, Frank B., and Remco C. Veltkamp. "3D face model fitting for recognition." European Conference on Computer Vision. Springer, Berlin, Heidelberg, 2008.

[28] Chang, Dah-Chung, and Wen-Rong Wu. "Image contrast enhancement based on a histogram transformation of local standard deviation." IEEE transactions on medical imaging 17.4 (1998): 518-531.

[29] Gander, Walter, Gene H. Golub, and Rolf Strebel. "Leastsquares fitting of circles and ellipses." BIT Numerical Mathematics 34.4 (1994): 558-578.

[30] http://www.astro.cornell.edu/research/projects/compressio n/entropy.html

[31] Willmott, Cort J.; Matsuura, Kenji (December 19, 2005). "Advantages of the mean absolute error (MAE) over the root mean square error (RMSE) in assessing average model performance". Climate Research. 30: 79-82.

[32] Li, Billy YL, et al. "Using kinect for face recognition under varying poses, expressions, illumination and disguise." Applications of Computer Vision (WACV), 2013 IEEE Workshop on. IEEE, 2013.

[33] Szwoch, Mariusz. "FEEDB: a multimodal database of facial expressions and emotions." Human System Interaction (HSI), 2013 The 6th International Conference on. IEEE, 2013.

[34] Hyndman, R. and Koehler A. (2005). "Another look at measures of forecast accuracy"

[35] Geng, Xin, et al. "Learning from facial aging patterns for automatic age estimation." Proceedings of the 14th ACM international conference on Multimedia. ACM, 2006.

[36] Hayashi, Jun-ichiro, et al. "Age and gender estimation from facial image processing." SICE 2002. Proceedings of the 41st SICE Annual Conference. Vol. 1. IEEE, 2002.

[37] Guo, Guodong, et al. "Image-based human age estimation by manifold learning and locally adjusted robust regression." IEEE Transactions on Image Processing 17.7 (2008): 1178-1188.

[38] Lanitis, Andreas, Chrisina Draganova, and Chris Christodoulou. "Comparing different classifiers for automatic age estimation." IEEE Transactions on Systems, Man, and Cybernetics, Part B (Cybernetics) 34.1 (2004): 621-628.

[39] Guo, Guodong, et al. "Human age estimation using bioinspired features." Computer Vision and Pattern Recognition, 2009. CVPR 2009. IEEE Conference on. IEEE, 2009.

[40] Yang, Guangzheng, and Thomas S. Huang. "Human face detection in a complex background." Pattern recognition 27.1 (1994): 53-63. 
[41] Wang, Jianguo, and Tieniu Tan. "A new face detection method based on shape information." Pattern Recognition Letters 21.6-7 (2000): 463-471.

[42] Wu, Haiyuan, Qian Chen, and Masahiko Yachida. "Face detection from color images using a fuzzy pattern matching method." IEEE Transactions on Pattern Analysis \& Machine Intelligence 6 (1999): 557-563.

[43] Hjelmås, Erik, and Boon Kee Low. "Face detection: A survey." Computer vision and image understanding 83.3 (2001): 236-274.

\section{Authors' Profiles}

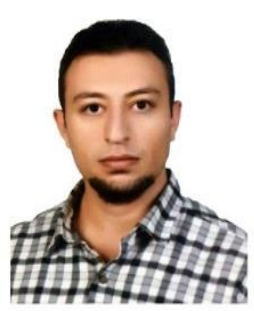

Seyed Muhammad Hossein Mousavi was born on July 30, 1990 at Tehran, Iran. He received his M.Sc. degrees from Bu-Ali Sina University, in 2017 in the branch of Artificial Intelligence. He had experience to be Pascal Programming TA in mentioned University. He has 14 published conference and journal papers so far. $\mathrm{He}$ is expert in machine vision, depth image processing, fuzzy logic evolutionary computation, Evolutionary art, OCR, NLP, data mining, expert systems and micro facial expressions recognition using RGBD images. Personal website: http://s-m-h-mousavi.ir/

How to cite this paper: Seyed Muhammad Hossein Mousavi, "A New Way to Age Estimation for RGB-D Images, based on a New Face Detection and Extraction Method for Depth Images", International Journal of Image, Graphics and Signal Processing(IJIGSP), Vol.10, No.11, pp. 10-18, 2018.DOI: 10.5815/ijigsp.2018.11.02 